Pacific Journal of Mathematics

HOMOTOPY COMPLEX PROJECTIVE SPACES WITH 


\title{
HOMOTOPY COMPLEX PROJECTIVE SPACES WITH DIVISIBLE SPLITTING INVARIANTS
}

\author{
ROBERT D. LITTLE
}

\begin{abstract}
A PL homotopy complex projective 4-space with integral splitting invariant $\sigma_{2}$ is smoothable if and only if $\sigma_{2} \equiv 0$ or $6(\bmod 14) . \mathbf{A}$ smooth homotopy complex projective 4-space with $\sigma_{2} \neq 0$ does not admit a smooth $Z_{3}$ action with a codimension 2 fixed submanifold.
\end{abstract}

1. Introduction. Let $X^{m}$ be a closed, simply connected PL $m$-manifold, where $m \geq 6$. The manifold $X^{m}$ is said to be smooth if its PL structure is compatible with a differentiable structure and almost smooth if it is smooth in the complement of a point. The purpose of this paper is to study these ideas in the special case where $X^{m}$ is a PL homotopy complex projective $n$-space, that is, $m=2 n, n \geq 3$, and $X^{2 n}$ is homotopy equivalent to $\mathbf{C} P^{n}$, complex projective $n$-space. The PL homeomorphism type of these manifolds can be described precisely in terms of numerical invariants. Let $P_{2 k}$ denote the simplyconnected surgery obstruction group for closed $2 k$-manifolds, $k \geq 2$, that is, $P_{2 k}=\mathbf{Z}$ if $k$ is even and $P_{2 k}=Z_{2}$ if $k$ is odd, [8]. A theorem of Sullivan, [8], asserts that the PL homeomorphism type of $X^{2 n}$ is determined by an $(n-2)$-tuple $\left(\sigma_{2}, \sigma_{3}, \ldots, \sigma_{n-1}\right)$ in $\prod_{k=2}^{n-1} P_{2 k}$. Sullivan suggested the problem of characterizing the smoothability or almost smoothability of these manifolds in terms of these splitting invariants. Every PL homotopy complex projective 3-space is smoothable, [10]. We obtain a complete answer in the case $n=4$ and in the cases $n=5$ or 6 , a complete answer modulo the value of the mod 2 invariant $\sigma_{3}$.

THEOREM 1.1. Every PL homotopy complex projective 4-space is almost smooth. A PL homotopy complex projective 4-space is smooth if and only if $\sigma_{2} \equiv 0$ or $6(\bmod 14)$.

THEOREM 1.2. A PL homotopy complex projective 5-space is almost smooth if and only if $\sigma_{4}-4 \sigma_{2}^{2}+10 \sigma_{2} \equiv 0(\bmod 28)$. There exists $a$ smooth homotopy complex projective 5-space with splitting invariants $\sigma_{2}$ and $\sigma_{4}$ if and only if $\sigma_{2}$ is even and $\sigma_{4}-4 \sigma_{2}^{2}+10 \sigma_{2} \equiv 0(\bmod 28)$. 
THEOREM 1.3. There exists an almost smooth homotopy complex projective 6-space with splitting invariants $\sigma_{2}$ and $\sigma_{4}$ if and only if $\sigma_{2}$ is even and $\sigma_{4}-4 \sigma_{2}^{2}+10 \sigma_{2} \equiv 0(\bmod 28)$. There exists a smooth homotopy complex projective 6-space with splitting invariants $\sigma_{2}$ and $\sigma_{4}$ if and only if $\sigma_{2}$ is even, $\sigma_{4}-4 \sigma_{2}^{2}+10 \sigma_{2} \equiv 0(\bmod 28)$, and

$$
-8 \sigma_{4} \sigma_{2}+\frac{64}{3} \sigma_{2}^{3}-4 \sigma_{4}+20 \sigma_{2}^{2}-\frac{421}{3} \sigma_{2} \equiv 0(\bmod 496) \text {. }
$$

We investigate certain finite group actions on homotopy complex projective spaces. Let $X^{2 n}$ be a $2 n$-manifold having the same integral cohomology ring as $\mathbf{C} P^{n}$ and let $p$ be an odd prime. Suppose $\mathbf{Z}_{p}$ acts on $X^{2 n}$ in such a way that the fixed point set of the action, $F\left(X^{2 n}\right)$, contains a codimension 2 submanifold $F_{0}$. It follows that $F\left(X^{2 n}\right)=$ $F_{0} \amalg$ point, ([3], p. 487). In [3], Dovermann has made a detailed study of the algebraic properties of such actions and has shown that they are often algebraically standard in a certain precise sense (see Definition 5.1). In particular, if $X^{2 n}$ admits an algebraically standard action, then the Pontrjagin class of $X^{2 n}$ must correspond to the Pontrjagin class of $\mathbf{C} P^{n}$. Dovermann, ([3], p. 490), raised the question of whether every $\mathbf{Z}_{3}$ action is algebraically standard in the case $n=4$, and we answer this question in the affirmative if $X^{8}$ is a PL homotopy complex projective 4-space.

THEOREM 1.4. If $X^{2 n}$ is a PL homotopy complex projective $n$-space with $n=4$ or 5 , then every locally linear $P L \mathbf{Z}_{3}$ action on $X^{2 n}$ with a codimension 2 fixed submanifold is algebraically standard.

This theorem settles the single open case of Dovermann's Theorem $\mathrm{A}(\mathrm{ii}),([3], p .488)$, and so the two results together imply that every smooth $\mathbf{Z}_{p}$ action of this type on a smooth homotopy complex projective 4-space is algebraically standard. If $\sigma_{2} \neq 0$, the Pontrjagin class of $X^{8}$ is not standard (Theorem 3.1), and so it follows that the exotic smooth homotopy complex projective 4-spaces of Theorem 1.1 with $\sigma_{2} \neq 0$ do not admit smooth $\mathbf{Z}_{p}$ actions with a codimension 2 fixed submanifold. Theorem 1.4 implies that Dovermann's Proposition 0.3 ([3], p. 489), is sharp at the prime $p=3$, and, if $d(p)\left(d^{\prime}(p)\right)$ is the smallest integer $n$ such that there exists a smooth (locally linear PL) non-standard action of this type on some smooth (PL) homotopy complex projective $n$ space, then Theorem 1.4, together with Dovermann's Proposition 0.3 
and Theorem 0.3 ([3], p. 489), imply that $d^{\prime}(3)=6$, and $6 \leq d(3) \leq$ 15.

The paper is organized as follows. In $\S 2$, we introduce notation and outline our general method of attack. Sections 3 and 4 contain the technical information necessary to implement the program outlined in $\S 2$. Section 3 contains a formula which relates the Pontrjagin class of a PL homotopy complex projective space to its integral splitting invariants. Section 4 contains a review of the results of Adams and Walker, [1], and Brumfiel, [2], on fibre homotopically trivial vector bundles over $\mathbf{C} P^{n}$ and the proofs of Theorems 1.1, 1.2, and 1.3. Section 5 contains the proof of Theorem 1.4.

2. Surgery problems and smoothability. Let $\mathrm{hPL}\left(\mathbf{C} P^{n}\right)$ and $\mathrm{hS}\left(\mathbf{C} P^{n}\right)$ be the sets of equivalence classes of homotopy triangulations and homotopy smoothings of $\mathbf{C} P^{n}$, respectively ([8], p. 19). If $\alpha_{n}: \mathrm{hS}\left(\mathbf{C} P^{n}\right)$ $\rightarrow \mathrm{hPL}\left(\mathbf{C} P^{n}\right)$ is the natural map and if $h: X^{2 n} \rightarrow \mathbf{C} P^{n}$ represents an element of $\mathrm{hPL}\left(\mathbf{C} P^{n}\right)$, then $X^{2 n}$ is smooth if and only if the equivalence class of $h$ is in the image of $\alpha_{n}$. If $\mathrm{hS}\left(\mathbf{C} P_{0}^{n}\right)$ is the set of equivalence classes of homotopy almost smoothings of $\mathrm{CP}^{n}$ ([2], p. 381), there is a natural map $\beta_{n}: \mathrm{hS}\left(\mathbf{C} P_{0}^{n}\right) \rightarrow \mathrm{hPL}\left(\mathbf{C} P^{n}\right)([8]$, p. 46) defined by the coning construction, and a general position argument at the singular point shows that if $h: X^{2 n} \rightarrow \mathbf{C} P^{n}$ represents an element of $\operatorname{hPL}\left(\mathbf{C} P^{n}\right)$, then $X^{2 n}$ is almost smooth if, and only if, the equivalence class of $h$ is in the image of $\beta_{n}$.

We study the mappings $\alpha_{n}$ and $\beta_{n}$ by studying the homomorphism $\gamma_{n}:\left[\mathbf{C} P^{n}, F / O\right] \rightarrow\left[\mathbf{C} P^{n} ; F / P L\right]$, which sends a smooth surgery problem with range $C P^{n}$ into the corresponding PL surgery problem. These maps are in a commutative diagram with exact rows ([8], p. 45).

0<smiles>[Tl]</smiles>

(2.1)

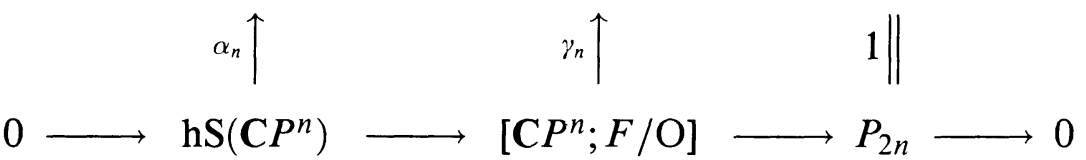

$[\mathbf{C P} ; F / \mathrm{PL}] \longrightarrow P_{2 n} \longrightarrow 0$

$[\mathrm{CP} ; F / \mathrm{O}] \longrightarrow P_{2 n} \longrightarrow 0$

The maps $\beta_{n}$ and $\gamma_{n-1}$ are related because there is a bijection $\operatorname{hPL}\left(X^{m}\right) \cong\left[X^{m}-\right.$ point; $\left.F / \operatorname{PL}\right]([8]$, p. 21$)$, and, if $X^{m}$ is almost smooth, there is a bijection $\mathrm{hS}\left(X_{0}^{m}\right) \cong\left[X_{0}^{m} ; \mathrm{F} / \mathrm{O}\right]$ ([2], p. 382). If 
$X^{m}=\mathbf{C} P^{n}$, we have the commutative diagram below:

$$
\begin{array}{cc}
\mathrm{hPL}\left(\mathbf{C} P^{n}\right) & \cong\left[\mathbf{C} P^{n-1} ; F / \mathrm{PL}\right] \\
\beta_{n} \uparrow & \gamma_{n-1} \uparrow \\
\mathrm{hS}\left(\mathbf{C} P_{0}^{n}\right) \stackrel{\cong}{\longrightarrow}\left[\mathbf{C} P^{n-1} ; F / \mathrm{O}\right]
\end{array}
$$

The group $\left[\mathbf{C} P^{n} ; F / \mathrm{PL}\right]$ can be described in terms of the splitting invariants. If $f: X^{2 n} \rightarrow \mathbf{C} P^{n}$ is a PL surgery problem and, if $k<n$, assume that $f$ is in general position with respect $\mathbf{C} P^{k} \subset \mathbf{C} P^{n}$. If $Y^{2 k}=f^{-1}\left(\mathbf{C} P^{k}\right)$, then the splitting invariant $\sigma_{k}$ in $P_{2 k}$ is defined to be the simply connected surgery obstruction to making $f \mid Y^{2 k}$ normally cobordant to a homotopy equivalence. Sullivan's theorem asserts that $\left[\mathbf{C} P^{n} ; F / \mathrm{PL}\right] \cong \prod_{k=2}^{n} P_{2 k}$, that is, a PL surgery problem is determined by an $(n-1)$-tuple $\left(\sigma_{2}, \sigma_{3}, \ldots, \sigma_{n}\right)$. The map $\left[\mathbf{C} P^{n} ; F / \mathrm{PL}\right] \rightarrow P_{2 n}$ sends a surgery problem into its obstruction, $\sigma_{n}$.

The homomorphism $\gamma_{n}$ is related to the homomorphism $\xi_{n}:\left[\mathbf{C} P^{n} ; F / O\right] \rightarrow k(n)$, where $k(n)$ is the kernel of the $J$-homomorphism, $J: \tilde{\mathrm{KO}}\left(\mathbf{C} P^{n}\right) \rightarrow J\left(\mathbf{C} P^{n}\right)$. This map is defined by Spivak uniqueness ([9], p. 105), which states that if $f: X^{2 n} \rightarrow \mathbf{C} P^{n}$ is a smooth surgery problem, then, in $\tilde{\mathrm{K}} \mathrm{O}\left(X^{2 n}\right)$, the tangent bundle of $X^{2 n}, \tau\left(X^{2 n}\right)$, satisfies the equation $\tau\left(X^{2 n}\right)=f^{*} \tau\left(\mathbf{C} P^{n}\right)+f^{*} \xi$, where $\xi$ is in $k(n)$. An element $\xi$ in $k(n)$ determines a smooth surgery problem, but not uniquely. In $\S 4$, we review the results of Adams and Walker, [1], and Brumfiel, [2], concerning $k(n)$, make some inferences concerning the image of $\gamma_{n}$ (and the images of $\alpha_{n}$ and $\beta_{n}$ via (2.1) and (2.2)), and prove Theorems $1.1,1.2$, and 1.3. In the next section, $\S 3$, we establish a formula relating the rational Pontrjagin class of a PL homotopy complex projective $n$-space and its integral splitting invariants. We will use this formula in the proof of Theorem 1.4 in $\S 5$.

3. The Pontrjagin class of a homotopy complex projective space. If $n \geq 3$, let $h: X^{2 n} \rightarrow \mathbf{C} P^{n}$ be a homotopy equivalence with splitting invariants $\left(\sigma_{2}, \sigma_{3}, \ldots, \sigma_{n-1}\right)$. The theorem below concerns the relationship between the Pontrjagin class $P_{i}\left(X^{2 n}\right)$ in $H^{4 i}\left(X^{2 n} ; \mathbf{Q}\right)$ and the integral splitting invariants. Let $y$ in $H^{2}\left(\mathbf{C} P^{n} ; \mathbf{Q}\right)$ be the generator of the cohomology algebra and let $\mathbf{Q}\left[x_{1}, x_{2}, \ldots, x_{i}\right], i \geq 0$, be the rational polynomial algebra on abstract generators, $x_{k}, 1 \leq k \leq i$, with the convention that if $i=0$, the symbol denotes $\mathbf{Q}$.

THEOREM 3.1. There exist universal polynomials $a_{i}$ in

$$
\mathbf{Q}\left[x_{1}, x_{2}, \ldots, x_{i}\right], \quad i \geq 0,
$$


such that $a_{0}=1, a_{i}(0,0, \ldots, 0)=0$, and $i \geq 1$, and, if $1 \leq i \leq[n / 2]$, then

$$
P_{i}\left(X^{2 n}\right)=\sum_{k=0}^{i}\left(\begin{array}{c}
n+1-k \\
i-k
\end{array}\right) a_{k}\left(\sigma_{2}, \sigma_{4}, \ldots, \sigma_{2 k}\right) h^{*} y^{2 l}
$$

LEMMA 3.3. Suppose that for fixed $i, 1<i \leq[n / 2], h$ is in general position with respect to $\mathbf{C} P^{2 i}$, and that formula (3.2) is valid for $P_{j}\left(X^{2 n}\right)$ if $1 \leq j \leq i-1$. Then $P_{j}\left(Y^{4 i}\right)=\rho_{j}\left(h \mid Y^{4 i}\right)^{*} y^{2 j}, 1 \leq j \leq i-1$, where

$$
\mu_{j}=\sum_{k=0}^{j}\left(\begin{array}{c}
2 i+1-k \\
j-k
\end{array}\right) a_{k}\left(\sigma_{2}, \sigma_{4}, \ldots, \sigma_{2 k}\right) .
$$

Proof of Lemma 3.3. Let $\zeta$ denote the canonical complex line bundle over $\mathbf{C P} \boldsymbol{P}^{m}, \mathbf{C} \boldsymbol{P}^{m} \subseteq \mathbf{C} \boldsymbol{P}^{n}$, if $m \leq n$. Let $\varepsilon_{0} \zeta$ be the real 2-plane bundle underlying $\zeta$, where $\varepsilon_{0}$ is the operator which assigns to a complex bundle the underlying real bundle ([5], p. 191). Since $h$ is in general position with respect to $\mathbf{C} P^{2 i}$, there is a direct sum decomposition of block bundles,

$$
\tau\left(X^{2 n}\right) \mid Y^{4 i}=\tau\left(Y^{4 i}\right) \oplus(n-2 i) h^{*}\left(\varepsilon_{0} \zeta\right),
$$

where $\tau\left(X^{2 n}\right)$ and $\tau\left(Y^{4 i}\right)$ denote the tangent block bundles of $X^{2 n}$ and $Y^{4 i}$, respectively.

Formula (3.5) and an inductive argument can be used to establish formula (3.4). The initial step, $j=1$ : the hypothesis concerning formula (3.2) and formula (3.5) imply that

$$
\left(n+1+a_{1}\left(\sigma_{2}\right)\right)\left(h \mid Y^{4 i}\right)^{*} y^{2}=P_{1}\left(Y^{4 i}\right)+(n-2 i)\left(h \mid Y^{4 i}\right)^{*} y^{2}
$$

since the Whitney sum formula holds over $\mathbf{Q}$. Therefore, $P_{1}\left(Y^{4 i}\right)=$ $\mu_{1}\left(h \mid Y^{4 i}\right)^{*} y^{2}$, where $\mu_{1}=2 i+1+a_{1}\left(\sigma_{2}\right)$. The inductive step: assume that (3.4) holds for all $j$ such that $1 \leq j<j_{0} \leq i-1$. To establish (3.4) at level $j_{0}$, note that the hypothesis about (3.2), (3.5), and the inductive hypothesis imply that $P_{j_{0}}\left(Y^{4 i}\right)=\rho_{j_{0}}\left(h \mid Y^{4 i}\right)^{*} y^{2 j_{0}}$, where

$$
\rho_{j}=\sum_{k=0}^{j_{0}}\left(\begin{array}{c}
n+1-k \\
j_{0}-k
\end{array}\right) a_{k}-\sum_{k=0}^{j_{0}-1}\left(\begin{array}{c}
n-2 i \\
j_{0}-k
\end{array}\right) \rho_{k} .
$$

If $c_{u}$ is the coefficient of $a_{u}$ in (3.6), $1 \leq u \leq j_{0}$, it follows that (3.4) at levels below $j_{0}$ implies that

$$
c_{u}=\left(\begin{array}{c}
2 i+1-u \\
j_{0}-u
\end{array}\right)
$$


To see this, note that the identity

$$
\left(\begin{array}{c}
a+b \\
c
\end{array}\right)=\sum_{i=0}^{c}\left(\begin{array}{l}
a \\
i
\end{array}\right)\left(\begin{array}{c}
b \\
c-i
\end{array}\right)
$$

can be used to show that the coefficient of $a_{u}$ in the second summation of $(3.6)$ is

$$
\left(\begin{array}{c}
n+1-u \\
j_{0}-u
\end{array}\right)-\left(\begin{array}{c}
2 i+1-u \\
j_{0}-u
\end{array}\right), \quad 0 \leq u \leq j_{0}-1
$$

Proof of Theorem 3.1. The proof is by induction and Lemma 3.3 will be used in the inductive step. Before starting the arguments, we remark that if one wishes to compute $P_{i}\left(X^{2 n}\right)$ as a rational multiple of $h^{*} y^{2 i}$ and $h$ is in general position with respect to $\mathbf{C} P^{2 i}$, it is enough to compute $P_{i}\left(X^{2 n}\right) \mid Y^{4 i}$ as a rational multiple of the cofundamental class of $Y^{4 i}$, since $h \mid Y^{4 i}$ has degree +1 .

The initial step, $i=1$ : put $h$ into general position with respect to $\mathbf{C} P^{2} \subset \mathbf{C} P^{n}$ and note that it follows from (3.5) at level $i=1$ that

$$
P_{1}\left(X^{2 n}\right) \mid Y^{4}=P_{1}\left(Y^{4}\right)+(n-2)\left(h \mid Y^{4}\right)^{*} y^{2} .
$$

Since $8 \sigma_{2}=L_{1}\left(P_{1}\left(Y^{4}\right)\right)\left[Y^{4}\right]-1$, where $L_{1}$ is the Hirzebruch $L$-class ([9], p. 162, [7], p. 225), and $\left(h \mid Y^{4}\right)^{*} y^{2}$ is the cofundamental class of $Y^{4}$, it is clear that (3.2) holds at level $i=1$ with $a_{1}\left(\sigma_{2}\right)=24 \sigma_{2}$. In particular, $P_{1}\left(X^{2 n}\right)$ is integral and determined by $\sigma_{2}$. The inductive step: assume that (3.2) holds for $P_{j}\left(X^{2 n}\right)$ for all $j$ such that $1 \leq j \leq$ $i-1$ and that $h$ is in general position with respect to $\mathbf{C} P^{2 i}$. Invoking Lemma 3.3 and using the definition of $\sigma_{2 i}$, we conclude that $P_{i}\left(Y^{4 i}\right)=$ $q_{i}\left(h \mid Y^{4 i}\right)^{*} y^{2 i}$, where $q_{i}$ is the rational polynomial expression in $\sigma_{2 k}$, $1 \leq k \leq i$, determined by (3.4) together with

$$
8 \sigma_{2 i}=L_{i}\left(P_{1}\left(Y^{4 i}\right), P_{2}\left(Y^{4 i}\right), \ldots, P_{i}\left(Y^{4 i}\right)\right)\left[Y^{4 i}\right]-1,
$$

where $L_{i}$ is the Hirzebruch $L$-class. The polynomial $a_{i}$ is defined by

$$
a_{i}=q_{i}-\sum_{k=0}^{i-1}\left(\begin{array}{c}
2 i+1-k \\
i-k
\end{array}\right) a_{k} .
$$

Since $h$ is in general position with respect to $C P^{2 i}$, we use (3.5) and conclude that

$$
P_{i}\left(X^{2 n}\right)=\left[q_{i}+\sum_{k=0}^{i-1}\left(\begin{array}{c}
n-2 i \\
i-k
\end{array}\right) \mu_{k}\right] h^{*} y^{2 i} .
$$


It follows from (3.4) and the identity

$$
\left(\begin{array}{c}
a+b \\
c
\end{array}\right)=\sum_{i=0}^{c}\left(\begin{array}{l}
a \\
i
\end{array}\right)\left(\begin{array}{c}
b \\
c-i
\end{array}\right)
$$

that if $c_{u}$ is the coefficient of $a_{u}$ in the summation in (3.9), $1 \leq u \leq$ $i-1$, then

$$
c_{u}=\left(\begin{array}{c}
n+1-u \\
i-u
\end{array}\right)-\left(\begin{array}{c}
2 i+1-u \\
i-u
\end{array}\right)
$$

and so (3.2) holds a level $i$ in view of (3.8).

We complete the proof of Theorem 3.1 by noting that $a_{i}(0,0, \ldots, 0)$ $=0, i \geq 1$, follows since $a_{1}(0)=0$ and, if $\sigma_{2 j}=0,1 \leq j \leq i$, then

$$
\mu_{j}=\left(\begin{array}{c}
2 i+1 \\
j
\end{array}\right), \quad 1 \leq j \leq i-1,
$$

by formula (3.4), if it is assumed, inductively, that $a_{j}(0,0, \ldots, 0)=0$, $1 \leq j \leq i-1$. This implies that $q_{i}=\left(\begin{array}{c}2 i+1 \\ i\end{array}\right)$ by naturality of the degree +1 map $h \mid Y^{4 i}$ and the fact $L_{i}\left[\mathbf{C} P^{2 i}\right]=1$. Thus $a_{i}(0,0, \ldots, 0)=0$ follows from (3.8).

We remark that if $n$ is even in Theorem 3.1, $n=2 i$, then $\sigma_{2 i}=0$ in formula (3.2). It follows easily from this remark and Theorem 3.1 that the Pontrjagin class of $X^{2 n}$ corresponds to the Pontrjagin class of CP $P^{n}$, that is, $P_{*}\left(X^{2 n}\right)=\left(1+h^{*} y^{2}\right)^{n+1}$ if and only if all the integral splitting invariants of $X^{2 n}$ vanish.

We conclude this section with some examples of the polynomials

$$
a_{i}: a_{1}\left(\sigma_{2}\right)=24 \sigma_{2}, \quad a_{2}\left(\sigma_{2}, \sigma_{4}\right)=\left(360 \sigma_{4}+576 \sigma_{2}^{2}-432 \sigma_{2}\right) / 7,
$$

and

$$
\begin{aligned}
a_{3}\left(\sigma_{2}, \sigma_{4}, \sigma_{6}\right)=(3780 / 31) \sigma_{6}+ & \left(56160 \sigma_{2} \sigma_{4}-6912 \sigma_{2}^{3}\right. \\
& \left.-39420 \sigma_{4}-57888 \sigma_{2}^{2}+13284 \sigma_{2}\right) / 217 .
\end{aligned}
$$

4. The kernel of the $J$-homomorphism. The reduced $K$-theory of $\mathbf{C} P^{n}, \tilde{\mathrm{K}} \mathrm{O}\left(\mathbf{C} \boldsymbol{P}^{n}\right)$, is a free abelian group with generators $\omega^{k}, k \geq 1$, where $\omega=\varepsilon_{0}(\zeta-1), \zeta$ is the canonical complex line bundle, and $\varepsilon_{0}$ is the realification operator $([1]$, Theorem 2.2$)$. There are relations which depend on $n(\bmod 4): \tilde{\mathrm{K}} \mathrm{O}\left(\mathbf{C} P^{2 i}\right)$ is free abelian with generators $\omega^{k}, 1 \leq k \leq i, \tilde{\mathrm{K}} \mathrm{O}\left(\mathbf{C} P^{4 i+3}\right)$ is free abelian with generators $\omega^{k}, 1 \leq$ $k \leq 2 i+1$, and $\tilde{\mathrm{KO}}\left(\mathbf{C} P^{4 i+1}\right)$ is free abelian with generators $\omega^{k}, 1 \leq$ $k \leq 2 i+1$, modulo the relation $2 \omega^{2 i+1}=0$.

Let $k(n)=\operatorname{kernel}\left\{J: \tilde{\mathrm{K} O}\left(\mathbf{C} P^{n}\right) \rightarrow J\left(\mathbf{C} P^{n}\right)\right\}$. Brumfiel ([2], pp. 400-401) has computed $k(n)$ for $n \leq 7$, and, if $u \in\left[C P^{n} ; F / O\right], n \leq 7$, 
expressed the integral splitting invariants of $u$ in terms of $\xi_{n}(u) \in k(n)$. We present Brumfiel's results with three very minor modifications. First, the integral splitting invariants as defined by formula (3.7) differ in sign from the splitting invariants in Brumfiel's paper. Secondly, note that it is not hard to see that if $\xi_{n}(u) \equiv 24 a \omega(\bmod$ higher powers of $\omega$ ), then $a=\sigma_{2}$. To see this, let $f: X^{2 n} \rightarrow \mathbf{C} P^{n}$ represent $u$, put $f$ into general position with respect to $\mathbf{C} P^{2}$ (where higher powers of $\omega$ are zero), and then compute $\sigma_{2}$ using the Hirzebruch index formula. Finally, we have done a little arithmetic with Brumfiel's formulas ([2], Lemma I.5), so that the formula for a given integral splitting invariant reflects the formulas for integral invariants of smaller subscript.

LeMmA 4.1(Brumfiel, [2], Lemmas I.4 and I.5). Let $\psi_{1}=24 \omega+$ $98 \omega^{2}+111 \omega^{3}, \psi_{2}=240 \omega^{2}+380 \omega^{3}$, and $\psi_{3}=504 \omega^{3}$. If $n \leq 7$, then $k(n)$ is generated by $\psi_{i}$ restricted to $\mathbf{C} P^{n}, i=1,2$, and 3 , and if $u \in\left[\mathbf{C} P^{n} ; F / O\right]$ with $\xi_{n}(u)=a \psi_{1}+b \psi_{2}+c \psi_{3}$, then

$$
\sigma_{2}=a \text {, }
$$

$$
\sigma_{4}=4 \sigma_{2}^{2}-10 \sigma_{2}-28 b
$$

$$
\sigma_{6}=8 \sigma_{4} \sigma_{2}-\frac{64}{3} \sigma_{2}^{3}+4 \sigma_{4}-20 \sigma_{2}^{2}+\frac{421}{3} \sigma_{2}+496(b+c) \text {. }
$$

Corollary 4.5. Suppose that $n \leq 7$ and that $v \in[\mathbf{C P} ; F / \mathrm{PL}]$ is determined by splitting invariants $\left(\sigma_{2}, \sigma_{3}, \ldots, \sigma_{n}\right)$. There exists an element $v^{\prime} \in\left[\mathbf{C P} P^{n} ; F / \mathrm{PL}\right]$ such that $v$ and $v^{\prime}$ have the same integral splitting invariants and $v^{\prime} \in$ image $\gamma_{n}$ if and only if the integral splitting invariants of $v$ satisfy the congruences,

$$
\sigma_{4}-4 \sigma_{2}^{2}+10 \sigma_{2} \equiv 0 \quad(\bmod 28),
$$

$$
\sigma_{6}-8 \sigma_{4} \sigma_{2}+\frac{64}{3} \sigma_{2}^{3}-4 \sigma_{4}+20 \sigma_{2}^{2}-\frac{421}{3} \sigma_{2} \equiv 0 \quad(\bmod 496) .
$$

Proof. Suppose that $v$ and $v^{\prime}$ have the same integral splitting invariants and $v^{\prime}=\gamma_{n}(u)$. If $\xi_{n}(u)=a \psi_{1}+b \psi_{2}+c \psi_{3}$, then formulas (4.2), (4.3), and (4.4) relate the integral splitting invariants of $v$ to $a, b$, and $c$, and so the congruences (4.6) and (4.7) hold. Conversely, suppose that the integral splitting invariants of $v$ satisfy the equations $\sigma_{4}-4 \sigma_{2}^{2}+10 \sigma_{2}=28 d$ and

$$
\sigma_{6}-8 \sigma_{4} \sigma_{2}+\frac{64}{3} \sigma_{2}^{3}-4 \sigma_{4}+20 \sigma_{2}^{2}-\frac{421}{3} \sigma_{2}=496 e \text {. }
$$


Let $u \in[\mathbf{C P} ; F / O]$ be an element such that $\xi_{n}(u)=\sigma_{2} \psi_{1}-d \psi_{2}+$ $(e+d) \psi_{3}$. It follows from Lemma 4.1 that the integral splitting invariants of $u$ are $\sigma_{2}, \sigma_{4}$, and $\sigma_{6}$ and so we may set $v^{\prime}=\gamma_{n}(u)$.

Proof of Theorem 1.1. The space PL/O is 6-connected ([7], p. 250), and so the tangent bundle of $X^{8}$-point lifts in the fibration PL/O $\rightarrow$ BSO $\rightarrow$ BSPL. Therefore, every PL homotopy complex projective 4-space is almost smooth. To verify the second statement in the theorem, note that it follows from Corollary 4.5 that if $v=\left(\sigma_{2}, \sigma_{3}, \sigma_{4}\right) \in$ $\left[\mathbf{C P} P^{4} ; F / \mathrm{PL}\right]$, then there exists $\sigma_{3}^{\prime} \in \mathbf{Z}_{2}$ such that $v^{\prime}=\left(\sigma_{2}, \sigma_{3}^{\prime}, \sigma_{4}\right) \in$ image $\gamma_{4}$ if and only if $\sigma_{4}-4 \sigma_{2}^{2}+10 \sigma_{2} \equiv 0(\bmod 28)$. Sullivan has remarked ([8], p. 45) that $(0,1,0) \in\left[\mathbf{C P} P^{4} ; F / P L\right]$ is in the image of $\gamma_{4}$. It follows from the properties of the universal $F /$ PL characteristic classes ([6], p. 80) that if $\left(\sigma_{2}, \sigma_{3}, \sigma_{4}\right)$ and $\left(\sigma_{2}^{\prime}, \sigma_{3}^{\prime}, \sigma_{4}^{\prime}\right)$ are two elements in the group $\left[\mathrm{CP} P^{4} ; F / \mathrm{PL}\right]$, then the group theoretic sum of the two elements is given by

$$
\left(\sigma_{2}, \sigma_{3}, \sigma_{4}\right)+\left(\sigma_{2}^{\prime}, \sigma_{3}^{\prime}, \sigma_{4}^{\prime}\right)=\left(\sigma_{2}+\sigma_{2}^{\prime}, \sigma_{3}+\sigma_{3}^{\prime}, \sigma_{4}+\sigma_{4}^{\prime}+8 \sigma_{2} \sigma_{2}^{\prime}\right) .
$$

In particular,

$$
\left(\sigma_{2}, \sigma_{3}, \sigma_{4}\right)+(0,1,0)=\left(\sigma_{2}, \sigma_{3}+1, \sigma_{4}\right) .
$$

It follows that if $\left(\sigma_{2}, \sigma_{3}^{\prime}, \sigma_{4}\right) \in$ image $\gamma_{4}$, then $\left(\sigma_{2}, \sigma_{3}^{\prime}+1, \sigma_{4}\right) \in$ image $\gamma_{4}$, and hence $\left(\sigma_{2}, \sigma_{3}, \sigma_{4}\right) \in$ image $\gamma_{4}$ if and only if $\sigma_{4}-4 \sigma_{2}^{2}+10 \sigma_{2} \equiv$ $0(\bmod 28)$. It follows that $\left(\sigma_{2}, \sigma_{3}, 0\right) \in$ image $\alpha_{4}$ if and only if $\sigma_{2} \equiv 0$ or $6(\bmod 14)$.

Proof of Theorem 1.2. The argument in the proof of Theorem 1.1 shows that if $\left(\sigma_{2}, \sigma_{3}, \sigma_{4}, 0\right) \in \operatorname{hPL}\left(\mathbf{C} P^{5}\right)$, then $\left(\sigma_{2}, \sigma_{3}, \sigma_{4}, 0\right) \in$ image $\gamma_{4}$ if and only if $\sigma_{4}-4 \sigma_{2}^{2}+10 \sigma_{2} \equiv 0(\bmod 28)$. This establishes the first assertion. To establish the second assertion, note that it follows from Corollary 4.5 and the fact that $\sigma_{2}(\bmod 2)$ determines the Arf invariant in the case $n=5$ ([2], p. 401, [4], p. 16) that there exists an element in $\left[\mathbf{C} P^{5} ; F / O\right]$ with splitting invariants $\sigma_{2}$ and $\sigma_{4}$, and with Arf invariant zero (i.e., $\sigma_{5}=0$ ), if, and only if, $\sigma_{2}$ is even and $\sigma_{4}-4 \sigma_{2}^{2}+10 \sigma_{2} \equiv 0(\bmod 28)$. In this situation, we have no control over $\sigma_{3} \in \mathbf{Z}_{2}$ because it is not clear that $(0,1,0,0) \in$ image $\gamma_{5}$.

Proof of Theorem 1.3. Theorem 1.3 follows from Corollary 4.5 in the same fashion as Theorems 1.1 and 1.2. We remark that smoothability or almost smoothability forces $\sigma_{5}=0([8], \mathrm{p} .45)$, and hence $\sigma_{2}$ is even 
in the statements in the theorem. We are unable to decide the value of $\sigma_{3}$ in this case just as in the case $n=5$.

5. Actions of $\mathbf{Z}_{3}$ with codimension two fixed point component. Let $X^{2 n}$ be a closed $2 n$-manifold with cohomology ring $\mathbf{Z}[y] /\left(y^{n+1}\right)$, where $y$ is a generator of $H^{2}\left(X^{2 n} ; \mathbf{Z}\right)$. Let $p$ be an odd prime and suppose that $\mathbf{Z}_{p}$ acts locally smoothly on $X^{2 n}$ in such a way that the fixed point set, $F\left(X^{2 n}\right)$, contains a codimension 2 submanifold, $F_{0}$. It follows that $F\left(X^{2 n}\right)=F_{0} \amalg$ point and, if $j: F_{0} \subset X^{2 n}$ and $y_{0}=j^{*} y$, then $H^{*}\left(F_{0} ; \mathbf{Z}\right)$ contains the polynomial ring $\mathrm{Z}\left[y_{0}\right] /\left(y_{0}^{n}\right)$ ([3], Corollary 0.1$)$. We use the notation $\tau$ for tangent bundle, $\nu$ for normal bundle, $c$ for Chern class, and $P_{*}$ for total Pontrjagin class. For an abelian group $H$, let $H_{f}=H /$ torsion denote its free part, and if $x \in H$, its image in $H_{f}$ is denoted by $f(x)$.

Definition 5.1 (Dovermann, [3]). An action on $X^{2 n}$ with codimension 2 fixed point component is algebraically standard if

(i) $P_{*}\left(X^{2 n}\right)=\left(1+y^{2}\right)^{n+1}$,

(ii) $H_{f}^{*}\left(F_{0} ; \mathbf{Z}\right)=\mathbf{Z}\left[f\left(y_{0}\right)\right] /\left(f\left(y_{0}\right)^{n}\right)$,

(iii) $f\left(P_{*}\left(F_{0}\right)\right)=\left(1+f\left(y_{0}\right)^{2}\right)^{n}$

(iv) $f\left(c\left(\nu\left(F_{0}, X^{2 n}\right)\right)\right)=1 \pm f\left(y_{0}\right)$,

(v) as a real representation, $\tau_{p t}\left(X^{2 n}\right)=n \nu_{x}\left(F_{0}, X^{2 n}\right)$, where $p t$ is the isolated fixed point and $x \in F_{0}$.

THEOREM 5.2. If $X^{2 n}$ is a PL homotopy complex projective $n$-space with $n=4$ or 5 , then every locally linear PL $\mathbf{Z}_{3}$ action on $X^{2 n}$ with a codimension 2 fixed submanifold is algebraically standard.

Proof. If $n=4, F_{0}$ is a 6-manifold and the normal block bundle, $\nu$, is a real 2-plane bundle ([3], p. 492). If $j_{!}: H^{k}\left(F_{0} ; \mathbf{Z}\right) \rightarrow H^{k+2}\left(X^{2 n} ; \mathbf{Z}\right)$ is the Gysin map, then $c_{1}(\nu)=j^{*} j_{!}\left(1_{F_{0}}\right)$ ([3], p. 503), and so there exists an integer, $r$, such that $f\left(c_{1}(\nu)\right)=r f\left(y_{0}\right)$. The block bundle equation $\tau\left(X^{8}\right) \mid F_{0}=\tau\left(F_{0}\right) \oplus \nu$ together with formula (3.2) in the case $n=4, i=1$ (recall that $a_{1}\left(\sigma_{2}\right)=24 \sigma_{2}$ ) yield the fact that $P_{1}\left(F_{0}\right)$ is integral and $f\left(P_{1}\left(F_{0}\right)\right)+f\left(P_{1}(\nu)\right)=\left(5+24 \sigma_{2}\right) f\left(y_{0}\right)^{2}$. Since $\nu$ is a real, orientable 2-plane bundle, $P_{1}(\nu)=c_{1}(\nu)^{2}$ ([7], p. 179), and so $f\left(P_{1}(\nu)\right)=r^{2} f\left(y_{0}\right)^{2}$. It follows that $f\left(P_{1}\left(F_{0}\right)\right)=s f\left(y_{0}\right)^{2}$, where

$$
r^{2}+s=5+24 \sigma_{2}
$$

Since the action is tame ([3], Theorem 1.1), we may apply the AtiyahSinger $G$-Signature Theorem. If the above formulas for the characteristic classes and the formula $f\left(y_{0}\right)^{3}\left[F_{0}\right]=r([3]$, Lemma 3.1) are 
put into the signature formula for $G=\mathbf{Z}_{3}$, ([3], p. 504), the resulting equation is

$$
r^{2}\left(2 r^{2}-s\right)= \pm 2
$$

Equations (5.3) and (5.4) imply that $r= \pm 1, s=4$, and $\sigma_{2}=0$. Conditions (iii) and (iv) follow immediately and formula (3.2) implies (i) since $\sigma_{2}=0$. Since $r= \pm 1, f\left(c_{1}(\nu)\right)^{3}=f\left(y_{0}\right)^{3}$ generates $H^{6}\left(F_{0} ; \mathbf{Z}\right)$ and so $f\left(y_{0}\right)$ generates $H^{*}\left(F_{0} ; \mathbf{Z}\right) /$ torsion ([3], Lemma 3.1), and this is (ii). We remark that ( $\mathrm{v})$ holds automatically in the case $p=3$ ([3], formula (1.4), p. 492).

The case $n=5$ is similar. The block bundle equation $\tau\left(X^{10}\right) \mid F_{0}=$ $\tau\left(F_{0}\right) \oplus \nu$ plus the equations $P_{1}(\nu)=c_{1}(\nu)^{2}, P_{i}(\nu)=0, i>2$ ([7], p. 174), together with formula (3.2) in the case $n=5, i=1,2$ (see the remarks at the end of the proof of Theorem 3.1 for $a_{1}\left(\sigma_{2}\right)$ and $\left.a_{2}\left(\sigma_{2}, \sigma_{4}\right)\right)$ imply that there are integers $r, s$ and $t$ such that $f\left(c_{1}(\nu)\right)=$ $r f\left(y_{0}\right), f\left(P_{1}\left(F_{0}\right)\right)=s f\left(y_{0}\right)^{2}$, and $f\left(7 P_{2}\left(F_{0}\right)\right)=t f\left(y_{0}\right)^{4}$. These integers satisfy the equations

$$
\begin{gathered}
r^{2}+s=6+24 \sigma_{2} \\
t+7 r^{2} s=105+360 \sigma_{4}+576 \sigma_{2}^{2}+408 \sigma_{2} .
\end{gathered}
$$

If the above formulas for the characteristic classes and the formula $f\left(y_{0}\right)^{4}\left[F_{0}\right]=r([3]$, Lemma 3.1), are put into the signature formula for $G=Z_{3}([3]$, p. 505), the resulting equation is

$$
r\left(60 r^{4}-20 r^{2} s+t-s^{2}\right)= \pm 5 .
$$

Formulas (5.5), (5.6), and (5.7) plus some elementary number theory show that $r= \pm 5$ is impossible. Therefore, $r= \pm 1$ and hence (iv) holds. We conclude also that (ii) holds, that is, $H_{f}^{*}\left(F_{0} ; \mathbf{Z}\right)=$ $\mathrm{Z}\left[f\left(y_{0}\right)\right] /\left(f\left(y_{0}\right)\right)^{5}([3]$, Lemma 3.1). In particular, the index of the 8 -manifold $F_{0}$ is 1 . It follows from the Hirzebruch Index Theorem and formulas (5.5) and (5.6) that $\left(t-s^{2}\right) / 45=1+8 \sigma_{4}=1$, that is, $\sigma_{4}=0$. If the information that $r= \pm 1$ and $\sigma_{4}=0$ is put into (5.7), the only possible conclusion is $\sigma_{2}=0$. Condition (i) now follows easily from formula (3.2). Since $s=5$ and $\sigma_{2}=\sigma_{4}=0, t=70$ and (iii) holds. As we remarked above, (v) holds automatically in the case $p=3$.

The author would like to thank Gregory Brumfiel and Karl Heinz Dovermann for help in the preparation of this paper. 


\section{REFERENCES}

[1] J. F. Adams and G. Walker, On complex Stiefel manifolds, Proc. Camb. Phil. Soc., 61 (1965), 81-103.

[2] G. Brumfiel, Homotopy equivalences of almost smooth manifolds, Comment. Math. Helv., 46 (1971), 381-407.

[3] K. H. Dovermann, Rigid cyclic group actions on cohomology projective spaces, Math. Proc. Camb. Phil. Soc., 101 (1987), 487-507.

[4] K. H. Dovermann, M. Masuda and R. Schultz, Conjugation involutions on homotopy complex projectives spaces, Japanese J. Math., 12 (1986), 1-35.

[5] D. Husemoller, Fibre Bundles, New York: McGraw-Hill, 1966.

[6] I. Madsen and R. J. Milgram, Classifying Spaces for Surgery and Cobordism of Manifolds, Ann. of Math. Studies, No. 92, Princeton University Press, Princeton, 1979.

[7] J. W. Milnor and J. D. Stasheff, Characteristic Classes, Ann. of Math. Studies, No. 76, 1974.

[8] D. Sullivan, Triangulated and smoothing homotopy equivalences and homeomorphisms, geometric topology seminar notes, Princeton University, 1967.

[9] C. T. C. Wall, Surgery on Compact Manifolds, London: Academic Press, 1970.

[10] Classification problems in differential topology V, On certain G-manifolds, Invent. Math., 1 (1966), 355-374.

Received March 1, 1988 and in revised form July 5, 1988.

University of Hawail at Manoa

HoNOlulu, HI 96822 


\section{PACIFIC JOURNAL OF MATHEMATICS EDITORS}

\author{
V. S. VARAdarajan \\ (Managing Editor) \\ University of California \\ Los Angeles, CA 90024-1555-05 \\ Herbert Clemens \\ University of Utah \\ Salt Lake City, UT 84112 \\ ThOMAS ENRIGHT \\ University of California, San Diego \\ La Jolla, CA 92093
}

R. FINN

Stanford University

Stanford, CA 94305

HeRmann FlaschKa

University of Arizona

Tucson, AZ 85721

VAUGHAN F. R. Jones

University of California

Berkeley, CA 94720

SteVen KercKhofF

Stanford University

Stanford, CA 94305

\section{ROBION KIRBY}

University of California

Berkeley, CA 94720

C. C. MOORE

University of California

Berkeley, CA 94720

HAROLD STARK

University of California, San Diego

La Jolla, CA 92093

\section{ASSOCIATE EDITORS}
R. Arens
E. F. BeCKenbaCH
B. H. NEUMANN
F. Wolf
K. YoshidA (1906-1982)

\section{SUPPORTING INSTITUTIONS}

UNIVERSITY OF ARIZONA

UNIVERSITY OF BRITISH COLUMBIA

CALIFORNIA INSTITUTE OF TECHNOLOGY

UNIVERSITY OF CALIFORNIA

MONTANA STATE UNIVERSITY

UNIVERSITY OF NEVADA, RENO

NEW MEXICO STATE UNIVERSITY OREGON STATE UNIVERSITY

\author{
UNIVERSITY OF OREGON \\ UNIVERSITY OF SOUTHERN CALIFORNIA \\ STANFORD UNIVERSITY \\ UNIVERSITY OF HAWAII \\ UNIVERSITY OF TOKYO \\ UNIVERSITY OF UTAH \\ WASHINGTON STATE UNIVERSITY \\ UNIVERSITY OF WASHINGTON
}

The Supporting Institutions listed above contribute to the cost of publication of this Journal, but they are not owners or publishers and have no responsibility for its content or policies.

Mathematical papers intended for publication in the Pacific Journal of Mathematics should be in typed form or offset-reproduced (not dittoed), double spaced with large margins. Please do not use built up fractions in the text of the manuscript. However, you may use them in the displayed equations. Underline Greek letters in red, German in green, and script in blue. The first paragraph must be capable of being used separately as a synopsis of the entire paper. In particular it should contain no bibliographic references. Please propose a heading for the odd numbered pages of less than 35 characters. Manuscripts, in triplicate, may be sent to any one of the editors. Please classify according to the scheme of Math. Reviews, Index to Vol. 39. Supply name and address of author to whom proofs should be sent. All other communications should be addressed to the managing editor, or Elaine Barth, University of California, Los Angeles, California 90024-1555-05.

There are page-charges associated with articles appearing in the Pacific Journal of Mathematics. These charges are expected to be paid by the author's University, Government Agency or Company. If the author or authors do not have access to such Institutional support these charges are waived. Single authors will receive 50 free reprints; joint authors will receive a total of 100 free reprints. Additional copies may be obtained at cost in multiples of 50 .

The Pacific Journal of Mathematics is issued monthly as of January 1966. Regular subscription rate: $\$ 190.00$ a year (5 Vols., 10 issues). Special rate: $\$ 95.00$ a year to individual members of supporting institutions.

Subscriptions, orders for numbers issued in the last three calendar years, and changes of address should be sent to Pacific Journal of Mathematics, P.O. Box 969, Carmel Valley, CA 93924, U.S.A. Old back numbers obtainable from Kraus Periodicals Co., Route 100, Millwood, NY 10546.

The Pacific Journal of Mathematics at P.O. Box 969, Carmel Valley, CA 93924 (ISSN 0030-8730) publishes 5 volumes per year. Application to mail at Second-class postage rates is pending at Carmel Valley, California, and additional mailing offices. Postmaster: send address changes to Pacific Journal of Mathematics, P.O. Box 969, Carmel Valley, CA 93924.

PUBLISHED BY PACIFIC JOURNAL OF MATHEMATICS, A NON-PROFIT CORPORATION Copyright (C) 1989 by Pacific Journal of Mathematics 


\section{Pacific Journal of Mathematics \\ Vol. 140, No. $2 \quad$ October, 1989}

Edoardo Ballico, Spanned and ample vector bundles with low Chern numbers

Marcy Mason Barge, Richard Swanson and Russell Bruce Walker,

Conjugacy class structure of smooth hyperbolic sectors . ........... 217

Jeffrey Stephen Fox, Adeles and the spectrum of compact nilmanifolds . . ..233

Robert D. Little, Homotopy complex projective spaces with divisible

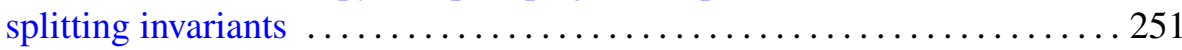

M. Scott Osborne and Garth William Warner, Jr., The Selberg trace formula. VII. Application of the truncation process to the continuous

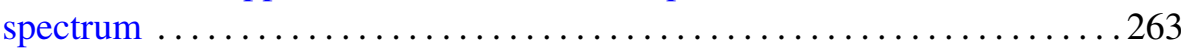

John R. Stembridge, On the eigenvalues of representations of reflection

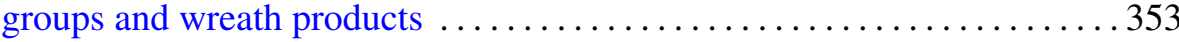

Ibrahim Salama, Corrections to: "Topological entropy and recurrence of

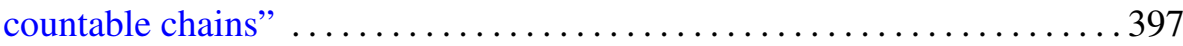

Robert Greene and Hung-Hsi Wu, Addendum to: "Lipschitz convergence of Riemannian manifolds" ............................... 398

Kayoko Shikishima-Tsuji, Correction to: "Galois theory of differential fields of positive characteristic" 